\title{
Visions of a more precise soil biology
}

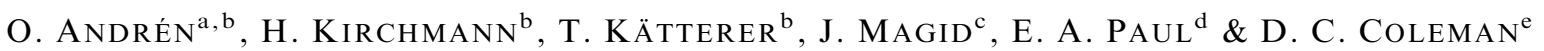 \\ ${ }^{\mathrm{a}}$ TSBF-CIAT, PO Box 30677-00100, Nairobi, Kenya, ${ }^{\mathrm{b}}$ Department of Soil Sciences, PO Box 7014, SLU, S-750 07 Uppsala, Sweden, \\ ${ }^{\mathrm{c}}$ The Royal Veterinary and Agricultural University, Bülowsvej 17, DK-1870 Frederiksberg C, Denmark, ${ }^{\mathrm{d}}$ NREL, Colorado State University, \\ Fort Collins, CO 80523-1499, USA, and ${ }^{\mathrm{e} I n s t i t u t e ~ o f ~ E c o l o g y, ~ E c o l o g y ~ A n n e x, ~ U n i v e r s i t y ~ o f ~ G e o r g i a, ~ A t h e n s, ~ G A ~ 30602-2360, ~ U S A ~}$
}

\begin{abstract}
Summary
Soils have often been viewed as a black box. Soil biology is difficult to study with the precision we would wish, due to the presence of considerable soil heterogeneity, a huge diversity of organisms, and a plethora of interacting processes taking place in a complex physical-chemical environment. We have isolated a tiny fraction of the known organisms, and the possible interactions of soil parent materials, landscape, land use, depth and time with the biota mean that we are to some extent still fumbling in the dark. There have been great advances, but we argue that the pace of advance could be faster. To progress, science needs new theory and concepts but also acceptable methodologies. Coherent and generally accepted theoretical knowledge exists in many areas, but there is a shortage of valid and exact methods to test new and sometimes even old hypotheses. New methods add knowledge, but they also can add to the confusion if they are not tied to the existing knowledge base. We speculate on how to improve soil biology through improving the way we perform and interpret research. Can we deal with soil variability? Can we measure the critical variables with adequate precision to test our hypotheses? Can we avoid reinventing the wheel? Can we find a balance between the freedom to test new and maybe even controversial ideas and the control and direction of research required by society?
\end{abstract}

\section{Introduction}

Soil science and soil biology have achieved many successes in their short period of existence, but one would always like to achieve more. Topical societal questions concerning global change, biodiversity, ecosystem services, sustainability and the need to provide food and fibre to a growing population while a significant proportion of our landscape is converted to biofuel production present opportunities, challenges and responsibilities. There is also a much larger audience for our field as soil biology is now included in general biology courses, including those in liberal arts colleges. This article is written by soil biologists and thus it focuses on what we know best, namely soil biology. Some of the challenges, however, are not unique to this subject. They may apply to all of soil science, and some even to most science.

Soil biology is in the midst of a major growth spurt involving many new researchers in both applied and fundamental studies. For example, a survey of 525 papers published between 1975 and 1999 on the biodiversity of bacteria and fungi in 10 of the most often cited journals showed that the number of papers published on rhizosphere-mycorrhiza increased from eight in 1975 to 80 in

Correspondence: O. Andrén. E-mail: olle.andren@mv.slu.se

Received 15 May 2007; revised version accepted 20 December 2007
1999 (Morris et al., 2002). The number of published papers on microbial habitats in soil increased from six to 60 per year during the same period. Interest in soil fauna has increased to a similar extent. This can be partly attributed to the international biodiversity treaty, which increased funding in numerous countries. The growing awareness of soil organisms as major players in global climate change and agricultural, forest and general environmental sustainability has also increased research opportunities, as have the breakthroughs in the molecular study of both soil fauna and microbiota.

One could claim that this has led and will continue to lead to major changes and even breakthroughs in our testing of concepts and hypotheses. There is also an increased interest in this field in many undergraduate institutions, and it has even been claimed that soils are the last (and greatest) frontier of ecology (Sugden et al., 2004). However, we argue that soil biology should and could make even greater contributions to soil science and science in general, considering the recent funding and interest.

Coleman (1985) wrote: 'Studies of ecological interactions in the soil require skilled usage of concepts from physics, chemistry, mathematics and biology'. More than 20 years have passed, but the argument that 'skilled usage' is the prime requisite for improving soil biology research still holds. We should not only 
adequately adopt concepts from other fields but also develop new concepts applicable to other fields. However, certain inherent properties of soils and biological systems (large heterogeneity, many levels of physical structures including micro-sites, the difficulty of making direct observations especially at depth, many different species that are difficult to extract and/or do not grow on ordinary laboratory media and the wide divergence of landscapes) make it difficult to perform scientific investigations with great precision. Experiments must be intelligently designed and carefully carried out and analysed, particularly in these exciting and challenging times in our field.

The difficulties involved in testing a hypothesis can even mean that 'well-known facts' that are simply wrong have a reasonable chance of surviving for a long time (see, e.g., Hedges et al., 2000). Less-than-perfectly executed science is not facing extinction. As readers and contributors to the scientific literature (including our fair share of less-than-perfect science), with more than 150 years of combined experience in this field, we will present and discuss some of the current challenges in soil biology (and, where appropriate, science in general). Where possible, we will suggest at least some partial solutions to the challenges - but often the solution is just improved awareness of the problem. This paper can hopefully serve to remind us of some common pitfalls that should be avoided. Fairly simple steps can usually be taken to ensure that errors, and more importantly flawed concepts, do not become part of our common understanding. Science can only progress if we dare to criticize what we do, even if we have done it for a long time.

Pointing out weaknesses can be a painful process and those who do so may become less than popular. For example, after publishing statistical analyses showing that the precision of projections from hydrological models may be less than previously thought, the author was accused of 'undermining the confidence of stakeholders and users of model predictions' and also of undermining the confidence in the science on which they are based (Beven, 2006). If this attitude is common also in soil biology, we look forward to interesting discussions after this paper is published.

\section{Current challenges in soil biology}

\section{Statistical analysis: precision and bias}

Problem. Good science should include a testable hypothesis (which could be a statement, an equation or even a simulation model) and a generally valid and exact test of this hypothesis (Popper, 1959/2000). This is not always easy in soil biology. Let us take a simple example (we use earthworms, for no other reason than that they are well known).

Hypothesis: earthworms increase decomposition rates of organic matter in the field. How do we test this hypothesis? We can extract all earthworms from plots (using electricity repeatedly, five replicates and trenches dug around to hinder invasion, etc.) and then compare decomposition rates with those in untreated plots (also with trenches dug around). We may find no significant differences during 3 years, possibly due to the old earthworm channels present. Perhaps we should continue the experiment for 10 years? Or perhaps our methods for measuring decomposition $\left(\mathrm{CO}_{2}\right.$ fluxes, soil carbon pool changes, etc.) are not sensitive enough to measure the small changes relative to the large amounts of heterogeneously distributed soil organic matter. We would only have a chance of detecting very large changes. We may obtain a statistically significant difference in 1 year, but one in the opposite direction the following year.

We also perform a laboratory experiment, where we add earthworms to homogenized soil cleared of worms. Now we obtain highly significant differences; if we scale the results up to the field level and project the effects over 30 years we find that earthworms have a major influence on decomposition rates (cf. Andrén et al., 2001). However, our field experiment did not indicate this, and neither did field experiments reported by other authors. In truth, we have not been able to really test the hypothesis, but when we publish we have at least added to our knowledge of earthworms. However, perhaps we only were able to publish the laboratory results, as a reviewer found the field results unexciting (see below). It is often the case that the complexity of process interactions in soils makes it difficult to obtain clear and unambiguous results in single experiments. Other good examples can be found in the study of mycorrhiza, food web interactions, the use of inoculants in biodynamic agriculture, and (sometimes) rhizobial inoculation. Naturally, there are sometimes good scientific reasons to believe that no consistent effects exist, but it is hard to believe that earthworms have absolutely no effect on decomposition rates of organic matter.

Continuing with the example of earthworms, one could argue that meta-analysis (mining the literature for reports on earthworms/decomposition and compiling the results) will provide a body of knowledge that taken together can be considered as a valid data set to test our hypothesis. However, such an analysis may give a very biased result in favour of the influence of worms, because the results in the published literature will be weighted towards those that showed a positive and significant effect. Authors, reviewers and editors tend to select for investigations that (i) have shown significant differences and (ii) show results pointing in the direction of their own knowledge (bias). The lack of significant effects seems to be less interesting, and most earthworm specialists think that earthworms are important. Note that using current knowledge to evaluate new results is exactly what a scientist should do; if an author claims that earthworms convert $\mathrm{Ca}$ to $\mathrm{N}$ we should be more sceptical (and demand solid proof; $\mathrm{cf}$. the rise and fall of cold fusion) than if they just report slightly increased $\mathrm{O}_{2}$ concentrations in a soil with earthworms. Here, we just point out that this evaluation will introduce a bias. Other areas of science than soil biology may have even greater problems with bias and statistical methods, as this quote from David Balding, Professor of Statistical Genetics, 
may indicate: 'The abysmal standard of statistical analysis in much of genetic epidemiology is little short of scandalous' (Ainsworth, 2007).

An example of the consequences of high variability and low awareness of statistics can be called the 'thesis syndrome'. Assume that a thesis is based on 200 statistical tests (treatment differences, correlations between variables, etc.), and that only some of the results are finally included in the thesis. If the $5 \%$ critical significance level is used, we can (roughly) expect $10(0.05 \times$ 200) significant differences in a randomized data set with no real treatment effects or correlations. In principle, this could lead to 10 significant differences reported from a data set consisting of random numbers. If this study was repeated, it would again yield 10 significant differences, but most probably not the same ones as before. In the real world, most scientists are aware of this problem of mass significance but its consequences are too often forgotten. Of course, if we apply the same reasoning to a long career as a scientist, we will sometimes obtain a type 1 error (finding differences where none exist), and sometimes a type 2 error (finding no differences where they do exist), even at the $1 \%$ or $0.1 \%$ significance levels. This is textbook knowledge, but it is sometimes forgotten when exciting new results seem to emerge or when there is excessive pressure for publications.

Solution. The conflicting results of the field and laboratory experiments on earthworms raise the question: are the differences due to the poor precision of the field measurements or are the mechanisms in the laboratory different from those in the field? To be able to answer this question we have to know the precision of the measurements and interpret our data accordingly. If the discrepancy persists, we can perhaps modify our laboratory set-up. In principle, improving precision is simple just do it by the book, while avoiding introducing bias. However, in the real world there are a number of obstacles. We do not always consult a skilled, practically inclined, statistician and listen to her or his advice before deciding on the final design of a project. Actually, statistics textbooks contain equations that can be used to calculate the necessary number of replicates for a given level of precision, when the variance and/or statistical distributions are known (a preliminary sampling yielding a fairly rough estimate will do). If your budget cannot cope with the necessary number of replicates, do something else. Do not sample three replicates because you cannot afford more and then hope for the best; perhaps it is possible to reduce the number of treatments, sites or soil types instead.

Good science tries to answer the most challenging question that one thinks is answerable with the techniques that are available. If the instrument resolution is too poor to resolve the variation existing in a process in nature, no statistical technique that one could apply to the results is of any value.

To reduce the bias introduced by the selection of positive results for publication, journals specialized in negative results are published within several branches of science, including, for example, ecology (Kotze et al., 2004). However, although this may have some effect it would be better to address the bias at the core, not try to balance one bias with another.

Avoiding mass significance, the 'thesis syndrome', should really not be a problem, once it is understood that if you carry out a lot of tests you will find more significant differences. However, in spite of numerous warnings and calls for improvement (e.g., Hurlbert, 1984; Kvålseth, 1985), the problem remains, and the current easy access to statistical packages may have made it worse. Good scientific journals give detailed instructions and urge authors to consult a statistician (see, e.g., Webster, 2001), but perhaps it is time for journals (and examination committees) to include a mandatory evaluation of statistical methods. We think it is more important for a scientific journal to publish reasonably solid and correct results than to be first with new revolutionary findings that later turn out to be products of flawed methodology. Finally, we must regrettably mention the wilful misinterpretation or even fabrication of results. This could probably be caught earlier by critical examination of the data and their statistical analysis, and more in-house peer review prior to publication.

\section{The method/concept gap}

Problem. Magid et al. (1997) coined the phrase 'modeling the measurable' but they also wrote 'and vice versa', while Elliott et al. (1996) wrote 'measuring the modelable'. This little wordplay concerns the critical relationship between models (or concepts) and measurements. One approach is to build a model of what is measured (e.g. soil carbon dynamics and daily weather) and exclude factors that are not. In its simplest form, this curve-fitting approach has little explanatory value and poor generality. In its more sophisticated form, based on sound theory and adapted to available (or at least measurable) data, this approach can be very useful. For example, the IPCC Tier 1 calculation methods for soil $\mathrm{C}$ pool changes, based on tabulated literature values (IPCC, 1995), or the slightly more complex five-parameter model describing soil $\mathrm{C}$ dynamics (Andrén \& Kätterer, 2001), do not necessarily yield less reliable predictions than more comprehensive models.

Another approach is to consider the detailed mechanisms (e.g. photosynthesis, microbial enzymatic reactions leading to decomposition of plant remains, food webs in the soil and how they affect the reactions, etc.) and their interactions in a complex and comprehensive conceptual model, which can be expressed as a large set of equations with many variables, parameters and interdependencies. This is a 'dictionary approach'; everything we know about the system is included. Most models are compromises between these two approaches.

The large number of parameters required by dictionary models increases the risk of 'equifinality', which occurs when the numbers of unknown parameters to optimize is large compared with the size of the data set available for calibration (Beven \& Freer, 2001). This means that for complex models, many possible different parameterizations are likely to match the observational data 
equally well from a statistical point of view, leading to additional uncertainty in predictions and/or extrapolation. Therefore, the analytical methods and available data are often not sufficient to critically test model concepts.

Models thus need data for calibration and validation (e.g. measured daily microbial biomass dynamics or the daily input of root-derived material and its chemical composition). The question then arises: can we measure this? The answer is usually: not really, but we can measure $x$, which is correlated to $y$. Thus we introduce another step: a calibration factor for conversion of $x$ to $y$. Ideally, this factor should have great precision and generality as well as no bias, but this is seldom the case. For example, the $k_{\mathrm{EN}}$ factor used to estimate nitrogen in chloroformextracted 'soil microbial biomass' (Jenkinson \& Powlson, 1976) has been shown to be highly variable (Joergensen \& Mueller, 1996). Despite this, only a small percentage of the publications in this subject area have derived their own calibration of the $k_{\mathrm{EN}}$ factor for the different soils under study.

Another example of the method/concept gap is that of the 'active' soil organic matter fractions used in soil carbon modelling. This is related to soil microorganisms and microbial products estimated by the chloroform fumigation technique (Jenkinson \& Powlson, 1976). In principle, we are given an easily measurable pool, clearly related to concepts central to our modelling efforts. But the discrepancies between the measurable changes in the estimates of the 'soil microbial biomass' pool and the concomitant changes in soil mineral nitrogen are often so large that it is difficult to retain this simple concept of the active fraction. Magid et al. (1996) examined the 'active' soil organic matter fractions by tracing ${ }^{14} \mathrm{C}$ from homogeneously labelled plant material through soils by means of various size and density fractionation methods. The added ${ }^{14} \mathrm{C}$-labelled plant material was preferentially utilized by the soil microorganisms, and thus the breakdown of native soil organic matter could not be estimated directly from these data. In order to isolate meaningful fractions, we have to tear up the fabric of the soil peds, and by doing so we run the risk of redistributing and cross-contaminating among the very classes that we conceptually define as distinct, thereby creating 'ghostly' artefacts as well as changing the physical nature of the soil. Thus, the 'active' fractions of native soil organic matter are probably distributed among particles of various size and density and the holy grail of an active fraction that can be isolated is likely to remain elusive.

The active fraction is probably best estimated in laboratory incubations in which the enzymes of the in situ microbial populations are allowed to decompose the organic matter. The active and slow fractions are then estimated by curve-fitting to the respiration data. The resistant fraction(s) is too refractory to be estimated in a standard laboratory incubation, and is often estimated by a chemical fractionation method such as acid hydrolysis. This leads to pool sizes and turnover rates that are analytically defined and which have relatively little inherent variability (Paul et al., 2006). The question remains however: to what extent can the results from a laboratory incubation of a disturbed sample be indicative of what happens in the field? There is the further challenge of relating laboratory incubation data measured at constant temperatures to the variable temperatures in the field. Recent results indicate that because of the inherent characteristics of activation energies derived from basic thermodynamics, increased temperatures may result in larger $Q_{10}$ coefficients for the resistant components of both litter inputs and native soil organic matter (Davidson \& Janssens, 2006; Conant et al., 2008). This would affect the interpretation of laboratory data and also has important implications for calculations of soil carbon dynamics under climate change.

Let us also examine how the precision of the measurements and the assumptions made can affect the precision of a calibration. We use an imaginary data set, but $y$ could be the number of earthworms obtained by careful sorting by hand, and $x$ could be those obtained by formalin extraction. Let us assume that we cannot afford a full programme of hand sorting, and use a linear function to convert the estimate obtained from formalin extraction to the 'true' number of earthworms (represented by hand sorting). Figure 1 (left) shows the data set, and the thick linear regression line. The coefficient of determination seems to be reasonable, with $58 \%$ of the variance
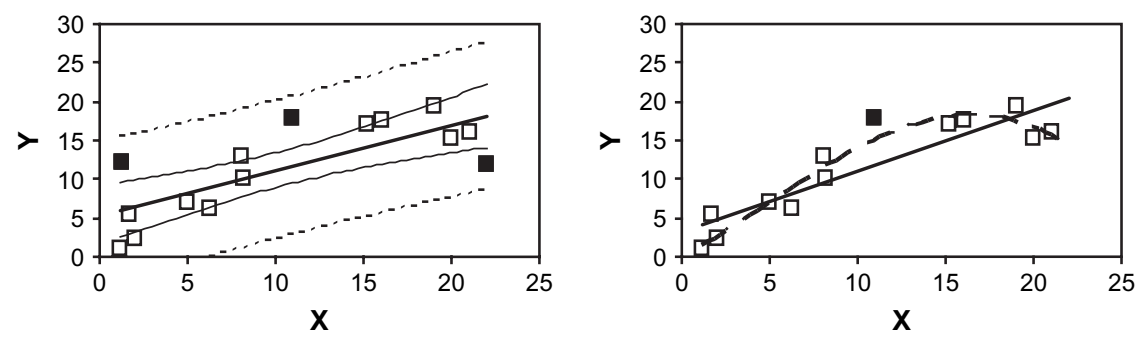

Figure 1 Left: Fifteen observations of a dependent variable $y$ plotted versus an independent variable $x$ and the corresponding regression line (-; $\left.R^{2}=0.58, P=0.00101\right)$ as well as $95 \%$ confidence intervals for the regression line (thin lines) and for individual predicted values (---). The three observations with the largest residuals correspond to the filled symbols. Right: The same observations but excluding two of the filled symbols as 'outliers' and linear $\left(R^{2}=0.78\right)$ and polynomial $\left(R^{2}=0.92\right)$ regression lines. 
explained by the model. Thus the correlation coefficient is as large as 0.76 , and the $P$ value indicates a highly significant regression. However, if we look at the $95 \%$ confidence intervals (stippled lines) for individual measurements we can see that an $x$ value of 10 has a $95 \%$ chance of predicting a value within the $y$ interval ranging from about 2 to 20 (draw a vertical line through $x=10$ and see where it intersects the confidence interval lines). We can also look at the regression line itself and the thin lines showing the $95 \%$ confidence limits for its position and slope. By moving the straight line up and down and changing its slopes within these limits we can see that, for example, an $x$ value of 0 will correspond to a $y$ value somewhere within the range 1-9.

Points that are distant from the regression line (possible outliers) can have a significant effect on the regression model and therefore, on its predictive ability. If such an observation is close to the mean value of $x$, but occupies an outlying position on the $y$-axis, its presence or absence will move the regression line up or down (bias) with only marginal effects on the slope. If such an influential observation is located towards one of the extreme ends of the $x$-axis, it will have a high leverage (i.e., it will mainly affect the slope of the regression). There are tests available for removing suspected outliers. According to one of these tests (Belsley et al., 1980), two observations in our data set were removed (Figure 1, right). Now, the $R^{2}$ of the regression line increases by $34 \%$ to 0.78 , and its slope increases by $35 \%$. However, the removal of these two outliers was based on the assumption that the relation between $x$ and $y$ was linear. If it is not, the removal of the 'outlier' at the upper end of the $x$-axis would not be justified. If we assume that the regression is not linear, but is best described by a polynomial, we obtain the stippled curve with a much better fit, $R^{2}=0.92$.

This exercise in data massage thus improved the fit considerably, but we are not sure we have come closer to the truth. Now we also have to deal with the confidence interval for the nonlinear model, which is beyond our scope here. One can also question whether we really did the right thing when we took the data points away? Further, an approximately normal distribution of the sample population is one of the prerequisites for applying parametric tests. The assumption of normality in our imaginary data set can be questioned due to its bi-modal distribution. Thus, the $F$-test and its resulting $P$ value as they were used here may not be relevant at all, or regression analysis may be inappropriate under such circumstances (Webster, 1989). There are methods to test for normality, and one can apply transformations of the input data to obtain normality, but we will stop here. In conclusion, too much is left open for interpretation when calibration data show large or even moderate variation (see Reichstein et al., 2005, for a typical example).

Another interesting example of how much the choice of model can influence the interpretation of a data set was recently presented by Gans et al. (2005), who re-analysed the data published by Sandaa et al. (1999). The data set contains information about community DNA from pristine and metalcontaminated soils. Gans et al. (2005) used a power law to describe the abundance distribution, which yielded quite different results to those originally reported. More than two orders of magnitude larger numbers of genomes were calculated to be present in soil, and the reduction in bacterial diversity due to heavy metal pollution increased from eightfold in the original analysis to a factor of 1000 . We do not have any views on who is 'right' and who is 'wrong' in this case, but this certainly illustrates how crucial the choice of statistical model can be.

Thus, the method/concept gap shows up in many ways, and when we use a method that yields an approximation of 'microbial biomass', 'mineral (or plant-available) nitrogen', 'protected carbon', 'soil carbon mass at steady-state' or 'oribatid abundance' it is too easy to forget that these are both dependent on the true value per se and the errors introduced by the physicochemical methods and models used (see, e.g., Six et al., 2000).

Solution. Decide why you want to use a model. If the purpose is practical, for example to calculate national or regional changes in soil carbon mass over a 30 -year period, it may be a good idea to be very simplistic and use crude approximations, even using linear approximations of processes that are inherently non-linear (IPCC, 1995). On the other hand, if the purpose is to increase our understanding of a system and generate new ideas for experiments, a very complex model using parameters of which some cannot be properly estimated and have to be tuned to make the model behave may be the best choice.

Improving the precision of calibration functions can be as simple as reducing their generality, that is using different parameter values or even functions for different cases (soil types, carbon content, etc.). However, in many cases, calibration functions may have only moderate precision despite reasonably large $R^{2}$ values, and then confidence limits should be given and their consequences for the results discussed, including possible bias.

A simple way to sustain awareness of the method/concept gap is to avoid using conceptual terms as far as possible when discussing results. For example, one can refer to a measurement as the 'heavy fraction' instead of 'protected', etc., but the end goal must always be to minimize the method/concept gap, preferably to zero.

\section{Paradigm shifts}

Problem. The idea behind classical humus analysis is to solubilise various fractions of humus to characterize soil organic matter by boiling, or extracting, soil in acids and bases. Early chemical analysis of both soil organic matter and its fractions led to the belief that the resistant components were very aromatic (Kononova, 1966). However, since the 1970s, NMR (nuclear magnetic resonance) and other less destructive methods have been used to investigate the structure of soil organic matter (Wilson, 1981). These methods indicate a large content of 
long-chain aliphatic and/or waxy groups and a smaller content of aromatics than the classical methods (Zech et al., 1985). Thus, a significant part of the aromatics reported from classical analysis probably was synthesized during analysis (Piccolo et al., 1999). There were some problems, though; the new methods were not fully quantitative, they were expensive and slow and difficult to interpret when applied to the soil milieu with its abundance of iron and other cations. We were introduced to a plethora of diagrams showing wavy lines lacking scale on at least one axis that were difficult for the average reader to interpret (see Hedges et al., 2000, for an example). Some researchers, inspired by these observations, claim that humus per se is a relatively low molecular weight substance and that the observed macromolecular structure can be explained as an aggregate of mixtures of various metal-cation complexes (Simpson et al., 2002).

It seems to be taking a long time to decide on what humus is and what properties it has. Then again, it might be futile to ask for a single definition of humus, as although the general structure of soil organic matter may be fairly constant across soil types with similar $\mathrm{C}: \mathrm{N}$ ratios, there are differences depending on the source of organic matter as well as the soil type (see, e.g., Hedges et al., 2000). The revision of how we view humus may, however, have been slowed down by the inertia that makes paradigm shifts so difficult (Kuhn, 1962); 'well-known facts' can survive for a long time, even when they are found to be at least not generally true.

Pathways of carbon uptake and translocation by mycorrhizal root systems are often complex, and simplistic assumptions about the sources of carbon for mycorrhiza may have to be abandoned. Many studies concerning nutrient cycling in soils have been based on hydroponic cultures, postulating the enhanced rate of uptake of key nutrients by the secretion of organic acids in mycorrhiza-free systems. It is essential to study plants, mycorrhiza and soils holistically to make significant advances in understanding of the mechanisms of enhanced mobilization and acquisition of many nutrients from soil or the external detoxification of metals (Jones et al., 2004). This might force us to rethink the validity of the simplifications of biology that are incorporated in nutrient and $\mathrm{C}$ flux models (Högberg \& Read, 2006).

The following example is a case in point. Measurements of ${ }^{13} \mathrm{C}$ in fungal sporocarps are useful for assessing mycorrhizal or saprotrophic status. Simultaneous measurement of ${ }^{14} \mathrm{C}$ in the sporocarps provides additional insight into mycorrhizal status. By measuring sporocarps, needles and litter from a site in Oregon, along with archived sporocarps, Hobbie et al. (2002) found clear separation of carbon sources for known mycorrhizal and saprotrophic fungi. The ${ }^{14} \mathrm{C}$ results for needles and mycorrhizal fungi indicated that small amounts of $\mathrm{C}$ may arise from stored carbohydrates, amino acids, organic nitrogen uptake and incorporation of soil-respired $\mathrm{CO}_{2}$ by anaplerotic (intermediates of the citric acid (TCA) cycle) or photosynthetic pathways.
It is quite understandable if soil biologists hesitate to embrace these results and revise our simple models, but this is possibly an area where more exact measurements may force us to abandon current simplifications.

Solution. The paradigm concept should not be emphasized too much, as it only reflects the tendency of humans to stick to what we think we know (particularly if it is our own invention) and should not be used as a critique of science in general. The scientific method, when properly used (strictly testing a hypothesis and accepting the outcome), should help considerably. However, the increased workload and productivity demands on scientists today may create a similar effect; you are too busy to take in new information and therefore stick to what you know. On the other hand, it is possible that some may be too eager to abandon the set of theories that we believe represent the truth, because new and revolutionary theories (or simply over-emphasizing the generality of your own observations) are perceived to be a way to fame and reputation. The solution to these problems, as well as to several other issues of scientific quality, is to allot more time to concept development and critical evaluation of all new results in relation to previously published literature.

\section{Reinventing the wheel: on forgotten literature}

Problem. Despite the critical view of meta-analysis expressed earlier in this paper, we certainly recommend more efforts in this direction. This can also be called critical reviewing. The accumulated mass of published papers is increasing at a frightening rate, but fortunately we can search, sort and download papers with great ease these days. However, nobody (at least none of the authors) can read and comprehend all the published literature in, for example, soil biology; this can only be achieved in narrower fields. Thus, critical reviews are extremely valuable and will become even more so. Perhaps the critical review and synthesis of a research area is the most valuable task a researcher can perform. This is not recognized enough. Wilson (1998) argued that the greatest challenge within science today is the accurate and complete description of complex systems (such as soils) and that there is a lack of attention paid to synthesizing established knowledge. We may add to our body of information, but often not to our understanding of this information.

Note that we are not claiming that good critical reviews and syntheses are lacking in the published literature; such works have been published recently (e.g. Bardgett et al., 2005; Coleman \& Whitman, 2005; Hooper et al., 2005). There is also an extensive and often excellent literature that is not downloadable, and perhaps not even found in searchable data bases. A surprisingly large proportion of results and ideas published in current papers can be found (at least in fairly similar forms) in earlier, pre-computer literature. There is nothing wrong with revisiting old territory, particularly when new methods are available. It would be preferable, though, if the modern visitors were aware of the pioneers, or at least of their maps of the 
territory. The readers of the modern paper can benefit greatly from reading early literature. Justice is also best served when the original progenitors of ideas are given adequate credit.

Let us give an example from soil biology. Two somewhat mathematically inclined soil scientists devised an analytical model of soil carbon dynamics (Andrén \& Kätterer, 1997). When the manuscript had been submitted, a French reviewer politely pointed out that most of the equations had been set up, solved and published in French by Hénin \& Dupuis (1945) and Hénin et al. (1959). If the authors of the 1997 paper had been aware of this pioneering work, they could have avoided reinventing the wheel, saved many days and nights of hard work, and perhaps produced a better paper.

Another example, this time from soil organic matter-tracer research, highlights both the importance of methodology and the need to refer to the pre-computer literature. Löhnis (1926) defined priming as the additional decomposition of native soil organic matter resulting from added substrate. This interesting concept could not be tested until the availability of tracers nearly 30 years later. Using tracers, Bingeman et al. (1953) showed that the added substrate truly caused an increased decomposition of native soil organic matter. After much discussion, this was accepted in the literature. Now, new techniques based on the availability of naturally occurring ${ }^{13} \mathrm{C}$ arising from switches between $\mathrm{C}_{3}$ and $\mathrm{C}_{4}$ photosynthetic plant types as well as enhanced $\mathrm{CO}_{2}$ concentration studies and the use of stable isotope probing are bringing in a group of young investigators who are not familiar with the detailed literature on priming from 50 years ago. The addition of a tracer allows one to measure both the tracer and the original soil $\mathrm{C}$ in the presence of added substrate from the tracer. Often, the added tracer results in a turnover, especially in the biomass where the tracer replaces the original $\mathrm{C}$ or $\mathrm{N}$ with an apparent, but not necessarily actual, loss of the soil constituent. This is not priming as originally defined, although this term has been used in this context. The recent review of this literature by Fontaine et al. (2004), who clearly redefined priming and cited the original literature, has not completely stopped this misuse. Priming can only be determined by combining the tracer study with a mass balance of the carbon contents in all the fractions to determine whether there has been a net loss of carbon, not just turnover.

Solution. We suggest that today's scientists need to allot more time to adequately reflect on and incorporate the results of previous studies and syntheses into their work. We also suggest that this is an area where scientific societies and academies could increase their efforts, perhaps by offering short-term scholarships for writing reviews, which besides being published could constitute excellent keynote presentations at conferences and workshops. Many scientific journals now publish reviews, and we encourage readers to write and submit manuscripts.

The lack of awareness of early literature is partly an educational problem, partly a question of accessibility. It should be easy for senior scientists and/or supervisors to put paper copies of selected early works in the hands of the students, and we are quite convinced these will enjoy and learn from the experience. They will not only be rewarded with new (or old) scientific insights, but probably also be baffled and amused by the differences in writing styles between now and then. Concerning availability, it is our hope that as much as possible of the ante .pdf literature will be scanned and become available in electronic form, or at least available as references in common scientific reference data bases. It is perhaps asking too much of the budding Google generation of soil biologists that they spend considerable time leafing through dog-eared index cards in stuffy libraries. The language barrier is a huge problem for those of us with limited skills in German, French, Russian, Japanese, or even Swedish and Danish; much of the early work was not published in English. Good translations to English, at least of benchmark papers, should be supported and read.

\section{Avoidable mistakes}

Problem. There are some fairly serious flaws in reasoning being published these days, as they have in the past. This is not at all bad for the progress of science; instead, the free presentation of ideas makes criticism and corrections possible. Fortunately, some flaws are easily discovered and disposed of, once a very basic understanding of systems and system boundaries is attained. Here are a few admittedly idiosyncratic examples, and we are convinced that every reader could add to the list.

Organic farming has been reported to give less leaching of nitrogen to groundwater than modern agriculture that uses commercial fertilizers. In one 6-year experiment 'organic' plots leached $34 \mathrm{~kg} \mathrm{~N} \mathrm{ha}^{-1}$ year $^{-1}$, and 'conventional' plots leached $38 \mathrm{~kg}$ (Torstensson et al., 2006). It may seem that the organic farming plots leached less, and put less strain on the environment. However, 'conventional' farming systems can include, for example, catch crops to mop up $\mathrm{N}$ during autumn, and these plots showed annual loads as small as $25 \mathrm{~kg} \mathrm{~N}^{-1}$ year $^{-1}$. Thus, 'conventional with catch crop' had the smallest leaching loads of the investigated systems in this study, but often only 'organic' and a not very clever 'conventional' cropping system are compared.

However, the main purpose of agriculture is to produce a vital product, for example, wheat grain. If a given amount of wheat is produced in a smaller area, the remaining area can be used for other purposes, for example biofuels, productive forests or natural reserves. Therefore, an equally rational unit for $\mathrm{N}$ leaching is amount leached per ton of crop yield. In the example above, 'organic' plots leached $17 \mathrm{~kg}$ nitrogen per ton of crop yield, 'conventional' leached $6.6 \mathrm{~kg}$ nitrogen per ton, and 'conventional with catch crop' leached $4.1 \mathrm{~kg} \mathrm{~N}$ per ton (Torstensson et al., 2006). Thus the context, or system boundary, heavily influences the result. We could also mention that system boundaries (in another sense) always have to be taken into account when discussing remedies to greenhouse gas emissions from soils; it is of little practical value to introduce measures 
that reduce $\mathrm{CO}_{2}$ emissions from a soil, if emissions of more potent greenhouse gases such as $\mathrm{CH}_{4}$ or $\mathrm{N}_{2} \mathrm{O}$ increase drastically (Robertson et al., 2000).

Another factor that must be considered is the difference between concentration and mass. Assume that we have a $30-\mathrm{cm}$ topsoil that is in balance with respect to $\mathrm{C}$ mass. The topsoil $\mathrm{C}$ pool is $10 \mathrm{~kg} \mathrm{~m}^{-2}$, the annual $\mathrm{C}$ input is $1 \mathrm{~kg} \mathrm{~m}^{-2}$, and $k$, the decomposition rate constant of soil organic $\mathrm{C}$ is 0.1 year $^{-1}$ (see, e.g., Andrén \& Kätterer, 2001). Now, assume that one plot is on a sandy soil (bulk density $1600 \mathrm{~kg} \mathrm{~m}^{-3}$ ) and another on a clay soil (bulk density $1200 \mathrm{~kg} \mathrm{~m}^{-3}$ ). Calculating the concentrations $\left(10 \mathrm{~kg} /\left(0.3 \mathrm{~m}^{3} \times 1600 \mathrm{~kg} \mathrm{~m}^{-3}\right)\right.$ for sand $)$ gives the following results for organic carbon content: sandy soil $2.1 \%$, clay soil $2.8 \%$. Thus, the observation that clay soils seem to protect soil $\mathrm{C}$ and have greater $\mathrm{C}$ concentrations (which are wrongly equated to more $\mathrm{C}$ mass) can be partly explained by bulk density differences.

Solution. It is tempting to state that it is easy to avoid the avoidable mistakes, but of course it is not. As pointed out elsewhere in this text, soil biologists must broaden their concepts and walk into unknown territory, where simple mistakes are often made: 'If we don't continuously extend our own scientific frontiers, we should not be surprised when others do it in a way we usually don't like' (Bouma, 2005). Thus, there are (and should be) many scientific disciplines involved, and nobody can master all of them. Cooperation is one keyword and for the examples given here, 'counting atoms' (i.e. calculating mass balances using the correct unit) is a good solution. Always be aware of the system boundaries, and try to reach at least a basic understanding of the models used in your work.

\section{On research funding}

Problem. Research performed at universities is usually funded by taxpayers, and the funds are not unlimited. There must be a selection process leading to the most efficient use of the available resources. There must also be a balance between 'academic' research mainly driven by curiosity and more 'goal-oriented' research. The first category is not intended to be immediately useful to the taxpayers right now, but it is considered necessary to make really groundbreaking discoveries possible. The second category is supposed to give results that are useful for society in the short term.

It has been pointed out that universities have undergone a 'second academic revolution' (Etkowitz, 2000), meaning that universities should become more useful to society as well as more entrepreneurial, and contribute to economic growth, solve acute environmental problems, etc. This leads to a shift from academic to goal-oriented research. As pointed out in the Introduction, soil biology has risen to this challenge, but the transition certainly has drawbacks. When the degree of influence of the donor on the individual scientist's agenda increases (i.e. the proportion of funding through short-term contracts increases), it affects how we do research. Scientists spend a large fraction of their time hunting for funding (perhaps with a $5 \%$ success rate), honing their skills in science, but particularly in writing applications. This is not a very efficient use of the time and scientific competence of highly skilled professionals.

Donors may also set up 'boundary conditions' that are not directly related to the scientific quality of the ideas proposed. For example, projects shall be: useful for solving problems in society; of a certain size, often large; spread between different institutions and countries; multi-disciplinary; address sociological, economic and gender issues, etc. Donors may argue that the decisions are still in the hands of the scientific community and based on scientific quality, because the applications are sent to scientists for peer review. However, the reviewers still have to judge by the more or less narrow criteria set up in the call for proposals, so the relative value of the research ideas per se for the final outcome may vary.

Multi- or trans-disciplinary research is not inherently bad (sometimes even preferable), but it does increase the time spent in administration and learning the basics of other disciplines. Social skills and good contact nets are valuable within such a system. In the words of Bernard Dixon, the former editor of New Scientist: 'Look around the institutions of science today and you'll find them headed by people who, whatever their record in research, are streetwise and assertive' (Dixon, 2007). Again, this is not necessarily bad, but scientific excellence is no longer the only, or perhaps even the most important, criterion for selection.

To stay in business, the researcher not only has to write good applications presenting exciting ideas (which is a good thing), but also has to zoom in on research areas formally or informally prioritized by donors. This leads to a funnelling of soil biologists into currently popular areas (e.g. biodiversity and C sequestration). Thus, international treaties and conventions channel research into directions that are decided at high-level meetings, and although these directions also depend on science, we should be aware of the fact that other factors such as national interests and the relative strength of lobby groups influence the outcome. Agenda 21, the 1992 document from the United Nations Conference on Environment and Development, highlighting biodiversity, is a good example. The ideas presented in this document have been very beneficial for soil biology as well as for biodiversity research in general. However, the document seems to postulate that biodiversity (not very clearly defined, but probably the number of species within, for example, an ecosystem) is 'important' for ecosystem functioning and that we should allocate funds to further the understanding of this importance (see also Andrén \& Balandreau, 1999). This has, of course, resulted in increased funding for research aimed at further proving the importance of biodiversity.

A recent, comprehensive consensus paper on biodiversity and ecosystem functioning reveals that more than a decade of research funding on biodiversity versus ecosystem function has led to few certain conclusions. Two examples: 
'3) The effects of species loss or changes in composition, and the mechanisms by which the effects manifest themselves, can differ among ecosystem properties, ecosystem types, and pathways of potential community change.

4) Some ecosystems properties are initially insensitive to species loss because (a) ecosystems may have multiple species that carry out similar functional roles, (b) some species may contribute relatively little to ecosystem properties, or (c) properties may be primarily controlled by abiotic environmental conditions.' (Hooper et al., 2005)

Even if the general benefits to ecosystem function of high biodiversity are not as great as postulated, our responsibility as humans to protect species from extinction is still the same, and research on how to avoid species extinction is crucial. Still, the questions should be asked: what if biodiversity per se is not so 'important'? If so, perhaps the efforts could have been better directed towards other areas of soil biological research?

The IPCC/Kyoto greenhouse gas reporting and mitigation agreement is another example of the channelling of resources due to signed conventions. For soil biology, this means that soil carbon pools, $\mathrm{CO}_{2}, \mathrm{~N}_{2} \mathrm{O}$ and $\mathrm{CH}_{4}$ emissions and the processes that control them receive attention and funding. Long-term experiments designed for other purposes are re-visited and used to calculate soil carbon dynamics, and eddy co-variation towers are being erected in most ecosystems. More or less sophisticated soil carbon models are being devised, calibrated and validated. After IPCC had realized that soils were major players, thousands of soil scientists all over the world have been redirected to research on greenhouse gases. This effort has yielded much valuable knowledge, and we can now make at least semi-quantitative projections of what will happen to soil carbon stocks if climate changes by $x$ or carbon input changes by $y$. The basic principles are well understood, although some very important details are debated (see, e.g., Reichstein et al., 2005; Conant et al., 2008 submitted). The 'only' serious problem remaining is the fairly poor precision in the estimates and projections.

However, the questions should be asked: Has the concentration of soil biology into areas demanded by society gone too far? Could it be that IPCC-generated reporting has led us too far towards book-keeping, that is calculating high-precision, 'accountable' changes in soil carbon pools, etc?

Solution. For the individual scientist or research group the short-term solution is to adapt to the situation, and try to be as successful as possible when applying for funds, maximizing the return per effort. A combination of experienced scientists with a good track record and knowledge of the history of the field with young scientists trained in new methodologies may be the most successful approach. The whole process of preparing and reviewing proposals that have a $5-10 \%$ chance of success is wasteful, and the use of short pre-proposals to limit the number of full-blown proposals a scientist has to work on can reduce the waste of time and effort for both those writing the proposals and the multitude of reviewers that must read them.
The degree of societal control of the scientist's agenda is continuously debated, and there is no single, perfect, solution. There is the question of how much money we should reserve for research and higher education, but perhaps the most important issue is how universities and research institutes are seen by society. Are we considered capable of setting the research agenda ourselves? The pendulum may have swung too far towards societal control, productivity demands and short term-funding, and if we are going to attract bright students that will become the next generation of soil biologists we will have to offer them considerable freedom to develop their own ideas and at least the possibility of a solid economic platform whilst pursuing a career in science.

\section{Concluding remarks}

The central problem that has to be solved for soil biology to move forward at an accelerating rate thus concerns the possibility to test hypotheses in a stringent way. The complexity of soil and soil organisms and its regional distribution makes it difficult to test hypotheses with a clear yes or no that is applicable to all areas of the world. There must be a way to reject a hypothesis. Without this we end up with idiosyncratic examples and not much new knowledge.

Soil biology has deep roots in applied agriculture and forestry, and much of agriculture's success has been empirically based. The adoption of practices depended on a certain level of success, not on a complete knowledge of the processes and organisms involved. Maybe the hypothesis being tested was: Will such a practice work in an economically viable way? If we had waited for a full theoretical explanation of how a certain practice increases the yield before applying it we would not have seen the huge increases in productivity and product quality in the middle of the last century. Only later was it found that some of the practices and particularly their misuse could be harmful to the environment, but overall the applied approach has been very successful.

Soil biology is now moving into many other areas and we can certainly supply answers to several of the major environmental questions of today. Careful forethought concerning the most important questions that can be answered with the available technology together with a good knowledge of past research into these hypotheses and questions will help open even further and faster the black box of soil biology. As we are so limited by the methods available, we also need to be heavily involved in the development of new technology that will not only help advance our field but also be of use in related areas.

\section{Acknowledgements}

N. Jarvis came up with good ideas, and L. Bergström helped us with leaching measurement data and gave valuable suggestions. A.-C. Hansson supplied valuable insights into the current debate on university versus society. Formas and Sida funded 
O. Andrén's stay at TSBF-CIAT, Nairobi. A number of editors and anonymous reviewers helped us to improve earlier versions of the manuscript.

\section{References}

Ainsworth, C. 2007. Let down by the statistics. Nature, 448, 849.

Andrén, O. \& Balandreau, J. 1999. Biodiversity and soil functioning from black box to can of worms? Applied Soil Ecology, 13, 105-108.

Andrén, O. \& Kätterer, T. 1997. ICBM: the introductory carbon balance model for exploration of soil carbon balances. Ecological Applications, 7, 1226-1236.

Andrén, O. \& Kätterer, T. 2001. Basic principles for soil carbon sequestration and calculating dynamic country-level balances including future scenarios. In: Assessment Methods for Soil Carbon (eds R. Lal, J.M. Kimble, R.F. Follett \& B.A. Stewart), pp. 495511. Lewis Publishers, Chelsea, MI.

Andrén, O., Kätterer, T. \& Hyvönen, R. 2001. Projecting soil fauna influence on long-term soil carbon balances from faunal exclusion experiments. Applied Soil Ecology, 18, 177-186.

Bardgett, R.D., Usher, M.B. \& Hopkins, D.W. (eds) 2005. Biological Diversity and Function in Soils. Cambridge University Press, Cambridge, UK.

Belsley, D.A., Kuh, E. \& Welsch, R.E. 1980. Regression Diagnostics. John Wiley \& Sons, Inc., New York.

Beven, K. 2006. On undermining the science? Hydrological Processes, 20, 3141-3146.

Beven, K. \& Freer, J. 2001. Equifinality, data assimilation, and uncertainty estimation in mechanistic modelling of complex environmental systems using the GLUE methodology. Journal of Hydrology, 249, 11-29.

Bingeman, C.W., Varner, J.E. \& Martin, W.P. 1953. The effect of the addition of organic materials on the decomposition of an organic soil. Soil Science Society of America Proceedings, 17, 34-38.

Bouma, J. 2005. Soil scientists in a changing world. Advances in Agronomy, 88, 67-96.

Coleman, D.C. 1985. Through a ped darkly: an ecological assessment of root-soil-microbial-faunal interactions. In: Ecological Interactions in Soil (eds A.H. Fitter, D. Atkinson, D.J. Read \& M.B. Usher). Special publication 4 of the British Ecological Society, pp. 1-21. Blackwell Scientific, Oxford.

Coleman, D.C. \& Whitman, W.B. 2005. Linking species richness, biodiversity and ecosystem function in soil systems. Pedobiologia, 49, 479-497.

Conant, R.T., Drijber, R.A., Haddix, M.L., Parton, W.J., Paul, E.A., Plante, A.F. et al. 2008. Sensitivity of organic matter decomposition to warming varies with its quality. Global Change Biology. doi: 10.1111/ j.1365-2486.2008.01541.x

Davidson, E.A. \& Janssens, I.A. 2006. Temperature sensitivity of soil carbon decomposition and feedbacks to climate change. Nature, 440, 165-173.

Dixon, B. 2007. What do we need to say to each other? New Scientist, 193, 2585, 46-47.

Elliott, E.T., Paustian, K. \& Frey, S.D. 1996. Modeling the measurable or measuring the modelable: a hierarchical approach to isolating meaningful soil organic matter fractions. In: Evaluation of Soil Organic Models Using Long-Term Datasets, Volume 38 (ed. D.S.
Powlson) NATO ASI Series I: Global Environmental Change, pp. 161-179. Springer-Verlag, Berlin, Heidelberg.

Etkowitz, H. 2000. The Second Academic Revolution: MIT and the Rise of Entrepreneurial Science. Gordon and Breach, London.

Fontaine, S., Bardoux, G., Benest, D., Verdier, B., Marriotti, A. \& Abbadie, L. 2004. Mechanism of the priming effect in a savannah soil amended with cellulose. Soil Science Society of America Journal, 68, 125-131.

Gans, J., Wolinsky, M. \& Dunbar, J. 2005. Computational improvements reveal great bacterial diversity and high metal toxicity in soil. Science, 309, 1387-1390.

Hedges, J.I., Eglinton, G., Hatcher, P.G., Kirchman, D.L., Arnosti, C., Derenne, S. et al. 2000. The molecularly-uncharacterized component of nonliving organic matter in natural environments. Organic Geochemistry, 31, 945-958.

Hénin, S. \& Dupuis, M. 1945. Essai de bilan de la matière organique du sol. Annales Agronomiques, 15, 17-29.

Hénin, S., Monnier, G. \& Turc, L. 1959. Un aspect de la dynamique des matières organiques du sol. Comptes rendus de l'Académie des Sciences (Paris), 248, 138-141.

Hobbie, E.A., Weber, N.S., Trappe, J.M. \& van Klinken, G.J. 2002. Using radiocarbon to determine the mycorrhizal status of fungi. New Phytologist, 156, 129-136.

Högberg, P. \& Read, D.J. 2006. Towards a more plant physiological perspective on soil ecology. Trends in Ecology and Evolution, 21, 548-554.

Hooper, D.U., Chapin, F.S., Ewel, J.J., Hector, A., Inchausti, P., Lavorel, S. et al. 2005. Effects of biodiversity on ecosystem functioning: a consensus of current knowledge. Ecological Monographs, 75, 3-35.

Hurlbert, S.H. 1984. Pseudoreplication and the design of ecological field experiments. Ecological Monographs, 54, 187-211.

IPCC 1995. IPCC Guidelines for National Greenhouse Gas Inventories: Workbook. IPCC, Bracknell.

Jenkinson, D.S. \& Powlson, D.S. 1976. The effects of biocidal treatments on metabolism in soil. 5. A method for measuring soil biomass. Soil Biology \& Biochemistry, 8, 209-213.

Joergensen, R.G. \& Mueller, T. 1996. The fumigation-extraction method to estimate soil microbial biomass: calibration of the $\mathrm{k}_{\mathrm{EN}}$ value. Soil Biology \& Biochemistry, 28, 33-37.

Jones, D.L., Hodge, A. \& Kuzyakov, Y. 2004. Plant and mycorrhizal regulation of rhizodeposition. New Phytologist, 163, 459-480.

Kononova, M.M. 1966. Soil Organic Matter. Pergamon, Oxford.

Kotze, D.J., Johnson, C.A., O'Hara, R.B., Vepsäläinen, K. \& Fowler, M.S. 2004. Editorial: the journal of negative results in ecology and evolutionary biology. Journal of Negative Results Ecology and Evolutionary Biology, 1, 1-5.

Kuhn, T. 1962. The Structure of Scientific Revolutions. The University of Chicago Press, Chicago.

Kvålseth, T.O. 1985. Cautionary note about $\mathrm{R}^{2}$. American Statistician, 39, 279-285.

Löhnis, F. 1926. Nitrogen availability of green manures. Soil Science, 22, 171-177.

Magid, J., Gorissen, A. \& Giller, K.E. 1996. In search of the elusive 'active' fraction of soil organic matter: three size - density fractionation methods for tracing the fate of homogeneously 14C-labelled plant materials. Soil Biology \& Biochemistry, 28, 89-99.

Magid, J., Mueller, T., Jensen, L.S. \& Nielsen, N.E. 1997. Modelling the measurable: interpretation of field-scale $\mathrm{CO}_{2}$ and $\mathrm{N}$-mineralization, 
soil microbial biomass and light fractions as indicators of oilseed rape, maize and barley straw decomposition. In: Driven by Nature: Plant Litter Quality and Decomposition (eds G. Cadish \& K.E. Giller), pp. 349-362. CABI Publishing, Wallingford, UK.

Morris, C.E., Bardin, M., Berge, O., Frey-Klett, P., Fromin, N., Girardin, H. et al. 2002. Microbial biodiversity: approaches to experimental design and hypothesis testing in primary scientific literature from 1975 to 1999. Microbiology and Molecular Biology Review, 66, 592-616.

Paul, E.A., Morris, S.J., Plante, A. \& Conant, R.T. 2006. Does the acid hydrolysis-incubation method measure meaningful soil organic carbon pools? Soil Science Society of America Journal, 70, 1023-1035.

Piccolo, A., Conte, P. \& Cozzolino, A. 1999. Effects of mineral and monocarboxylic acids on the molecular association of dissolved humic substances. European Journal of Soil Science, 50, 687-694.

Popper, K. 2000 (first published 1959). The Logic of Scientific Discovery. TJ International Ltd, Padstow, Cornwall.

Reichstein, M., Kätterer, T., Andrén, O., Ciais, P., Schulze, E.-D., Cramer, W. et al. 2005. Temperature sensitivity of decomposition in relation to soil organic matter pools: critique and outlook. Biogeosciences, 2, 317-321.

Robertson, G.P., Paul, E.A. \& Harwood, R.R. 2000. Greenhouse gases in intensive agriculture: contributions of individual gases to the radiative forcing of the atmosphere. Science, 289, 1922-1925.

Sandaa, R.-A., Torsvik, V., Enger, Ø., Daae, F.L., Castberg, T. \& Hahn, D. 1999. Analysis of bacterial communities in heavy metal- contaminated soils at different levels of resolution. FEMS Microbiology Ecology, 30, 237-251.

Simpson, A.J., Kinery, W.L., Hayes, M.H.B., Spraul, M., Humpfer, E., Dvortsak, P. et al. 2002. Molecular structures and associations of humic substances in the terrestrial environment. Naturwissenschaften, 89, 84-88.

Six, J., Merckx, R., Kimpe, K., Paustian, K. \& Elliott, E.T. 2000. A re-evaluation of the enriched labile soil organic matter fraction. European Journal of Soil Science, 51, 283-293.

Sugden, A., Stone, R. \& Ash, C. 2004. Ecology in the underworld. Introduction. Science, 304, 1613.

Torstensson, G., Aronsson, H. \& Bergström, L. 2006. Nutrient use efficiencies and leaching of organic and conventional cropping systems in Sweden. Agronomy Journal, 98, 603-615.

Webster, R. 1989. Is regression what you really want? Soil Use and Management, 5, 47-53.

Webster, R. 2001. Statistics to support soil research and their presentation. European Journal of Soil Science, 52, 331-340.

Wilson, M.A. 1981. Applications of nuclear magnetic resonance spectroscopy to the study of the structure of soil organic matter. Journal of Soil Science, 32, 167-186.

Wilson, E.O. 1998. Consilience - The Unity of Knowledge. Little Brown and Company, London.

Zech, W., Kögel, I., Zucker, A. \& Alt, H. 1985. CP-MAS- ${ }^{13}$ C-NMRSpektren organischer Lagen einer Tangelrendzina. Zeitschrift für Pflanzenernährung und Bodenkunde, 148, 481-488. 